\title{
Susceptibility of Acanthamoeba to multipurpose lens-cleaning solutions
}

\author{
Ana Paula Costa de Aguiar ${ }^{1 *}$, Caroline de Oliveira Silveira ${ }^{1}$, \\ Mari Aline Todero Winck ${ }^{1}$ and Marilise Brittes Rott ${ }^{*}$
}

${ }^{1}$ Departamento de Microbiologia, Imunologia e Parasitologia, Instituto de Ciências Básicas da Saúde.

Universidade Federal do Rio Grande do Sul, Porto Alegre, RS, Brazil

\begin{abstract}
The present study investigated the susceptibility of Acanthamoeba spp. trophozoites to two multipurpose systems for cleaning and maintenance of contact lenses. Three strains of trophozoites from the ATCC (A. castellani T4, A. castellani Neff, and A. polyphaga) and two Acanthamoeba isolates obtained from swimming pools (PT5 and PO1) were placed in monoxenic culture. To test their survival in cleaning solutions for contact lenses, the trophozoites were exposed for 4 and $24 \mathrm{~h}$ to two multipurpose solutions (A and B), and were then inoculated into a new monoxenic culture. Amoebic growth on the plates was observed after $72 \mathrm{~h}$ of incubation. Trophozoites from all three ATCC strains and one isolate from a swimming pool (PO1) grew in all plates after $4 \mathrm{~h}$ of exposure to solutions A and B. After $24 \mathrm{~h}$, the ATCC strains and the PO1 isolate showed growth in most of the plates treated. Only the PT5 isolate showed susceptibility to both solutions over the time intervals tested. The two solutions were not completely effective against most strains and isolates over the time intervals tested. These results are important, since species of Acanthamoeba are widely distributed in the environment and are potential agents of eye pathologies.
\end{abstract}

\section{Keywords}

Acanthamoeba, multipurpose solutions, keratitis

\section{Introduction}

Species of the genus Acanthamoeba are amphizoic free-living amoebae, widely distributed in the environment. The clinical importance of these organisms is due to the fact that they are responsible for cases of human infections, such as encephalitis in immunocompromised patients, and amoebic keratitis in immunocompetent patients, especially users of contact lenses.

Historically, Acanthamoeba keratitis (AK) has been associated with corneal trauma and exposure to contaminated water (Cohen et al. 1985, Theodore et al. 1985). With the growing use of contact lenses, the incidence of AK has increased, and although this has not been precisely determined, it has been estimated to affect 1.65 to 2.09 per million contact-lens wearers in the United States (Schaumberg et al. 1998) and 3.06 per million in The Netherlands (Morlet et al. 1997). The disease is a potentially severe eye infection caused by progressive inflammation and ulceration in the cornea (Zheng et al. 2004), and symptoms include intense pain, photophobia, decreased vision, tearing, and swelling (Marciano-Cabral and Cabral 2003). If during the early stage, inappropriate treatment is instituted, the agent can penetrate deeply into the cornea and other parts of the eye (McClellan et al. 1987). Thus, Acanthamoeba can invade tissues and settle in the stroma, causing severe infections that can lead to the enucleation of the ocular globe (Khan et al. 2002).

The virulence of the amoeba is related to its genotype, which is determined by using the $18 \mathrm{~S}$ rDNA gene sequence. Evolutionary studies have led to the identification of at least 16 genotypes (T1-T16) based on rRNA gene sequencing (Stothard et al. 1998; Gast et al. 2001; Booton et al. 2005; Maghsood et al. 2005; Corsaro et al. 2010). Most cases of keratitis are associated with genotype T4 (Yu et al. 2004, Molmeret et al. 2005).

Although the pathogenesis of AK is not well recognized, the use of contact lenses has been a major risk factor (Zheng et al. 2004). Current studies show that over $90 \%$ of cases occur in contact-lens wearers. Predisposing factors for keratitis in users of contact lenses are the use of non-sterile solutions, swimming in a pool while wearing contact lenses, and inadequate disinfection of contact lenses (Radfort et al. 2002). 
Therefore, considering the growing number of wearers of both corrective and cosmetic contact lenses, and the increasing incidence of infections associated with their use, the purpose of this study was to determine the efficacy of two multipurpose systems that are currently used in Brazil, against ATCC strains and isolates of Acanthamoeba spp.

\section{Materials and Methods}

Two disinfecting and maintenance solutions for contact lenses (designated $\mathrm{A}$ and $\mathrm{B}$ ), chosen based on their dominance in the market and registered in ANVISA (BRASIL 2011) in the category „health products“, and a commercial saline solution, which served as a negative control, were evaluated against three ATCC strains (Acanthamoeba castellani T4, Acanthamoeba castellani Neff, and Acanthamoeba polyphaga) and two environmental isolates from swimming pools (PT5 and PO1) (Caumo and Rott 2011). ). The genotype to which each belongs and its source are listed in Table I.

The trophozoites of Acanthamoeba spp. were grown in a $50 \mu \mathrm{L}$ aliquot of PYG (De Carli and Moura 2007) liquid medium $(2 \%$ proteose peptone, $0.2 \%$ yeast extract, and $1.8 \%$ glucose) supplemented with penicillin $\mathrm{G}$ potassium $(400 \mathrm{IU} / \mathrm{ml})$ and streptomycin $(400 \mathrm{~mL} / \mathrm{mL})$. Aliquots containing approximately 100 trophozoites were added to the surface of $1.5 \%$ non-nutrient agar (NNA) (De Carli and Moura 2007), previously coated with a suspension of heat-killed Escherichia coli. The plates were sealed with Parafilm ${ }^{\circledR}$ and incubated at $30^{\circ} \mathrm{C}$ for $72 \mathrm{~h}$. Areas of plates with a confluent amount of trophozoites were delimited by a $2 \times 2 \mathrm{~cm}$ square ( 300 trophozoites/square).

Subsequently, the plates were opened under sterile conditions, and the squares were cut and transferred to $50-\mathrm{ml}$ aliquots of the A, B, and saline solutions. All tests were performed in triplicate. After 4 and $24 \mathrm{~h}$ of exposure to the test solutions at room temperature, the viability of the trophozoites was tested. Each square, after being removed from the A, B, and saline solutions, was transferred to Page saline solution (De Carli and Moura 2007) for $5 \mathrm{~min}$ in order to rinse the squares. Then, the squares were individually placed face down in a monoxenic culture and the plate was sealed with Parafilm. The amoebic growth on plates was inspected after $72 \mathrm{~h}$ incubation at $30^{\circ} \mathrm{C}$, under a light microscope (100X). Ten random fields were observed, and the number of trophozoites in each field was counted. The mean number of amoebae per field was calculated.

Table I. List of genotypes and sources of the Acanthamoeba strains and isolates investigated

\begin{tabular}{lccc}
\hline Name & ATCC & Genotype & Source \\
\hline Acanthamoeba castellani T4 & ATCC30010 & T4 & Cornea with keratitis \\
Acanthamoeba castellani Neff & ATCC50492 & T4 & Soil \\
Acanthamoeba polyphaga & ATCC 30461 & T4 & Cornea with keratitis \\
Isolate PT5 & - & T5 & Pool water \\
Isolate PO1 & - & T4 & Pool water \\
\hline
\end{tabular}

Table II. Growth of Acanthamoeba castellani T4, Acanthamoeba castellani Neff, and Acanthamoeba polyphaga strains, and isolates PT5 and PO1 after $4 \mathrm{~h}$ and $24 \mathrm{~h}$ contact with solutions A and B

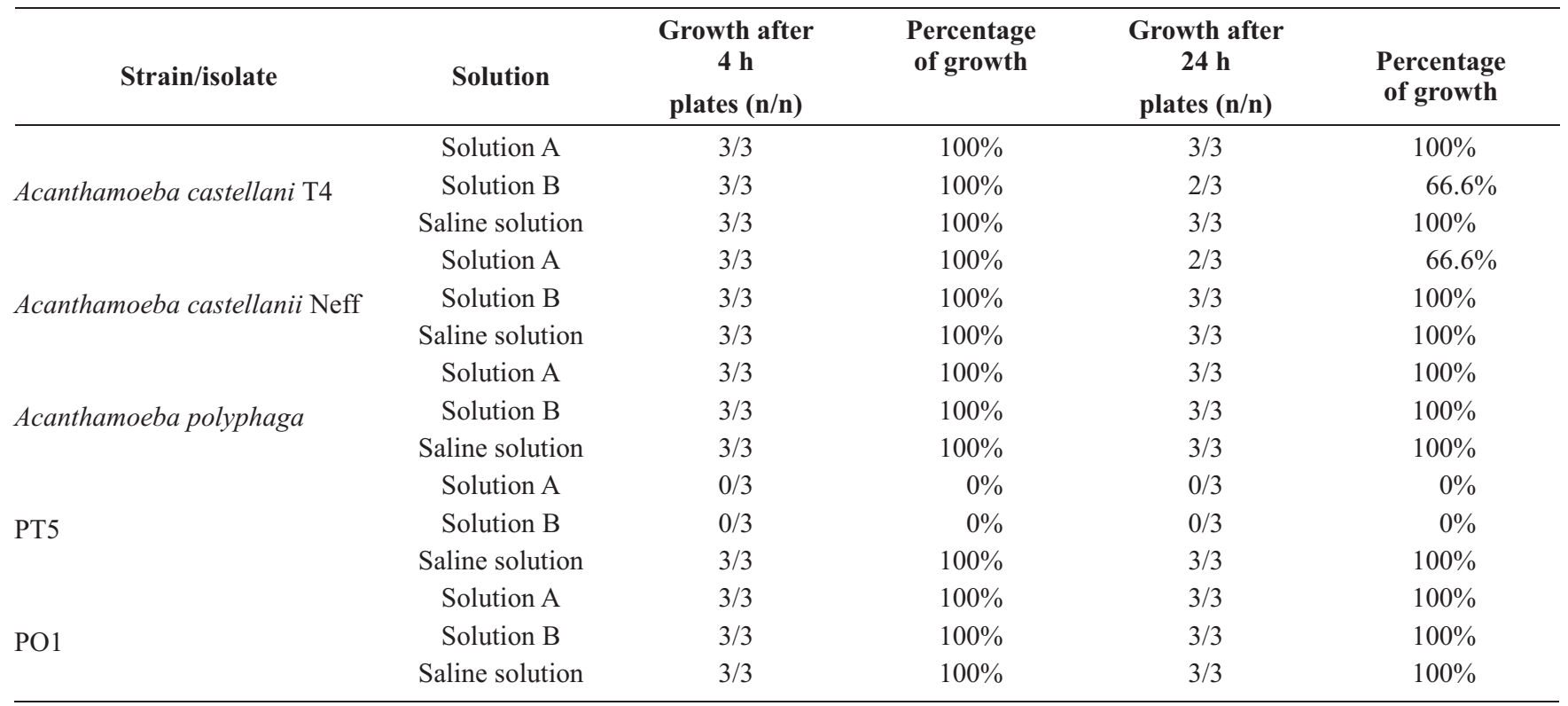




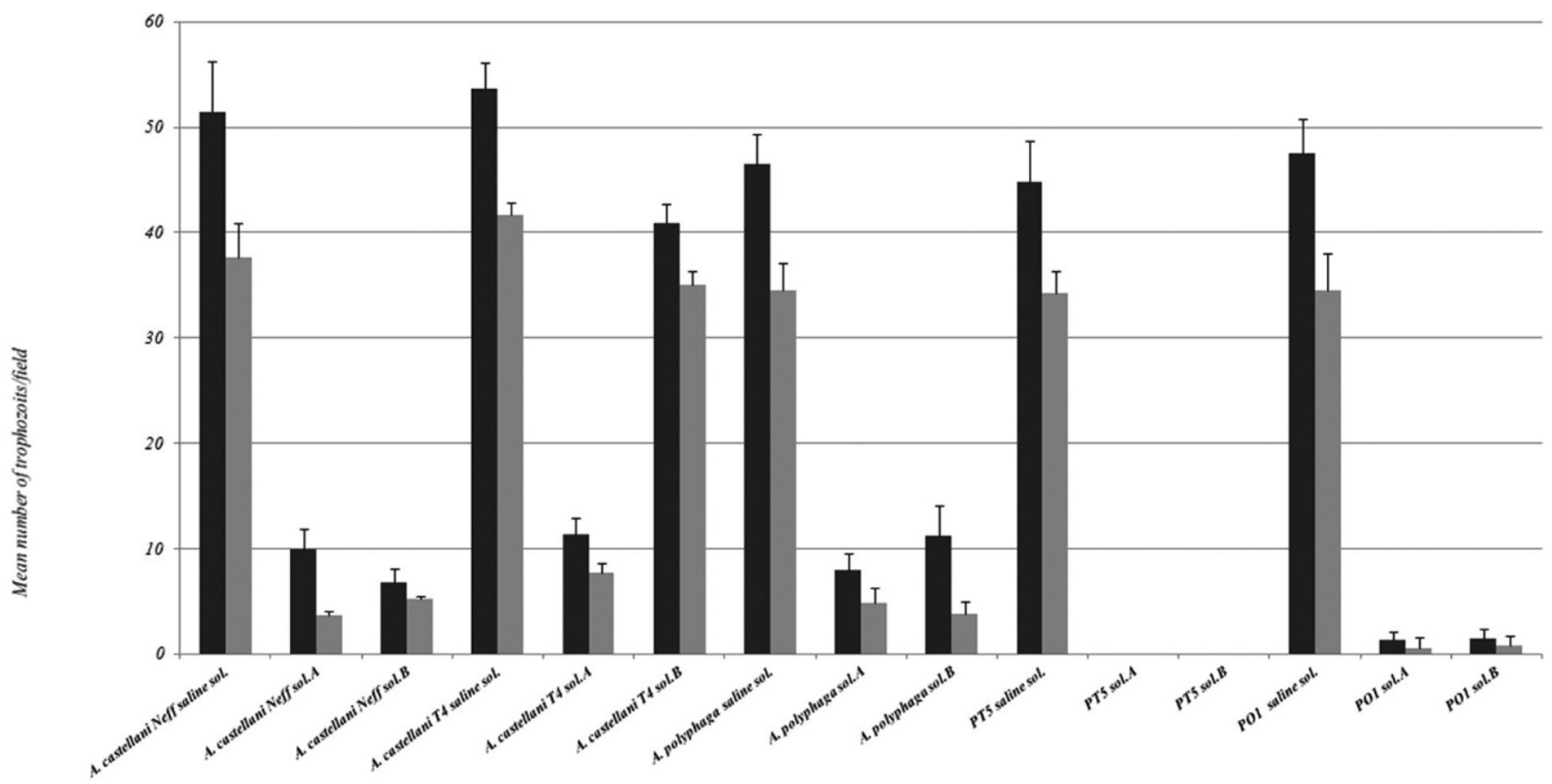

Fig. 1. Number of trophozoites per field after treatment with solutions A and B and a saline solution (negative control), time intervals tested for Acanthamoeba castellani Neff, Acathamoeba castellani T4, Acanthamoeba polyphaga, and isolates PT5 and PO1. Data represent mean \pm standard deviation

\section{Results}

The two solutions evaluated did not kill off all the amoebae of ATCC strains and the swimming-pool isolate (PO1) of Acanthamoeba after $4 \mathrm{~h}$ of immersion of the NNA blocks containing protozoan trophozoites, in triplicate testing. The Acanthamoeba castellani Neff strain showed growth in all plates after $24 \mathrm{~h}$ contact with solution B, and in two of three plates after $24 \mathrm{~h}$ contact with solution A. Acanthamoeba castellani T4 grew in all plates after $24 \mathrm{~h}$ in contact with solution A, and in two of three plates after $24 \mathrm{~h}$ with solution B. Acanthamoeba polyphaga and isolate PO1 grew in all plates after $24 \mathrm{~h}$ of contact with solutions A and B. PT5 isolate showed no growth in solutions A and B over both time intervals tested (4 and 24h) when inspected after $72 \mathrm{~h}$ of incubation. Then these plates were following until the tenth day of the incubation and no growth was observed. The results are presented in Table II.

Analyzing the average growth per field, all strains and isolates showed a higher growth rate when in contact with the saline solution, in comparison to the multi-purpose solutions. Comparing the contact periods, the organisms that were in contact for $24 \mathrm{~h}$ had a lower growth rate than those that remained for $4 \mathrm{~h}$ in all solutions. The exception was strain PT5, which showed no growth in either time interval.

Regarding the efficacy of the solutions, $A$. castellani strain T4 grew more slowly in solution A than in solution B. The other isolates showed no significant differences in growth in the two solutions. The results are shown in Figure 1.

\section{Discussion}

Acanthamoeba is a widely distributed organism in the environment, and is commonly found in anthropogenic aquatic environments. Its resistant form tolerates adverse environmental conditions, allowing it to survive in the various physical and chemical treatments, and arousing the interest of many researchers in the study of this amphizoic protozoan. Carlesso et al. (2007) isolated and characterized amoebae of the genus Acanthamoeba and Naegleria in biofilms and dust, from a hospital in southern Brazil. In this study, the most prevalent genus found was Acanthamoeba. Caumo et al. (2009), evaluating the presence of Acanthamoeba in swimming pools, found that some water samples studied were positive for freeliving amoebae, all identified as belonging to the genus Acanthamoeba. The characterization of Acanthamoeba isolates by thermal and osmotolerance assays indicated that some of the isolates were potentially pathogenic. Pens et al. (2008), evaluating the presence of bacteria and Acanthamoeba spp. in the biofilm and storage liquid of contact-lens cases of volunteers also from a hospital in southern Brazil, found positive samples. In this study, younger users of contact lenses showed more contamination in their contact-lens cases.

In our study, only isolate PT5, which belongs to the T5 genotype, showed susceptibility to the multipurpose solutions. However, studies comparing the T4 and T5 genotypes for resistance to multipurpose solutions, curiously, showed that the T5 genotype has a higher resistance (Hiti et al. 2002; Shoff et al. 2007). Only isolates of the T2, T3, T4, T6, T11 (Walo- 
chnick et al. 2000; De Jonckheere 2003) and T5 (albeit rarely) (Spanakos et al. 2006; Iovieno et al. 2010) genotypes have been associated with keratitis. The T4 genotype is found in $90 \%$ of the cases of AK and is the most prevalent genotype found in clinical and environmental samples (Yu et al. 2004; Molmeret et al. 2005).

In the present study, we observed that the two solutions studied were not completely effective against most strains and isolates over the time intervals tested, and only a swimmingpool isolate (PT5) showed susceptibility. This shows that the recommendation by the product manufacturers that it is possible to dispense with rubbing the lens with the fingers when multipurpose solutions are used for cleaning, is questionable. As discussed in a previous study (Lipener and Ray 2008), several factors can contribute to infections in contact-lens wearers, such as poor maintenance and inadequate hygiene, including in this case by eliminating the digital-rubbing step. In another study (Shoff et al. 2008), Acanthamoeba trophozoites of different origins, belonging to the T4 genotype, were tested against the hydrogen-peroxide systems and the multipurpose solutions. The results demonstrated the ineffectiveness of multipurpose solutions against Acanthamoeba strains, and the two hydrogen-peroxide systems tested were much more efficient. In 2007, the Centers for Disease Control and Prevention (CDC) started a study of 22 national centers for ophthalmology in the United States, to assess whether the possible increase in the number of AK cases found in central Illinois, was also occurring in other U.S. centers. Data received from 13 centers also demonstrated an increase in cases of AK, and the investigation revealed a relationship between cases of keratitis and the use of Complete MoisturePlus multipurpose solution. At that time the manufacturer undertook a voluntary recall of the product (Bryan et al. 2010).

In another study (Patel and Hammersmith 2008), a literature review of infections in contact lenses and their relationship to the multi-purpose solutions was performed, which indicated a rise in infections caused by Acanthamoeba and Fusarium fungus. Moreover, it was found that multipurpose solutions are more effective against bacteria than against fungi and Acanthamoeba, and that these formulas are not as effective under real-world conditions.

However, it is very important to inform users about the risks caused by the substitution of solutions for cleaning and disinfection by saline, which does not clean or remove protein deposits from lenses, leaving them dirtier, contaminated, and less comfortable. Moreover, the saline environment can be conducive to the proliferation of microorganisms that can affect eye health. Another problem is crystallization of the serum, in other words, salt (sodium chloride) crystallizes and adheres to the lenses, and may cause discomfort and reduce their useful life. Indeed, the major cause of toxic reactions in contact-lens users is the use of saline, because of the presence of chemical preservatives in its composition (Lipener and Ray 2008). However, a large number of people prefer physiological solutions than other cleaning systems, because of their affordable cost.
The results of this study are similar to those found by most authors who have studied the effectiveness of disinfection and preservation solutions for contact lenses against Acanthamoeba. The incidence of Acanthamoeba keratitis has been increasing in recent years, related to the higher frequency of contact-lens use, so it is important that the use, cleaning, handling, and storage are done with medical supervision, since with proper maintenance, the risk of developing eye disorders will be much lower. As for manufacturers, larger studies are needed regarding the efficacy of multipurpose solutions against Acanthamoeba spp., thus increasing the quality and reliability of their products, and perhaps the return of the recommendation for including digital rubbing in the contact-lens cleaning step.

Acknowledgements. The authors thank the Microbiology, Immunology and Parasitology Department of the Universidade Federal do Rio Grande do Sul, Professor Karin da S. Caumo for their assistance, and Dr. Janet W. Reid for revising the English text.

\section{References}

Booton G.C., Visvesvara G.S., Byers T.J., Byers T.J., Kelly D.J., Fuerst P.A. 2005. Identification and distribution of Acanthamoeba species genotypes associated with nonkeratitis infections. Journal of Clinical Microbiology, 43, 1689-1693. DOI: 10.1128/JCM.43.4.1689-1693.2005.

BRASIL, 2011. Agência Nacional de Vigilância Sanitária. Serviço de Registro de Produtos para Saúde [ANVISA web site]. Available in: www.anvisa.gov.br. Accessed November 7, 2010.

Bryan K., Bugant J., Chang T., Chen S., Rosenberg J., Hammond R., McConnell K., Sanderson R., Nakata M., Wakida C., Austin C., Bestudik J., Bordson M.G., Conover C., Granzow L., Rea V., Chu A., Luckman E., Signs K., Harper J., Damrow T., Mosher E., Kruger K., Saheli E., Cassidy M., Hatch J., Weltman A., Garcia Rivera E.J., Garcia Y., Kainer M.A., Archer J., Joslin C., Cernoch P., Jones D., Hamill M., Matoba A., Pflugfelder S., Wilhelmus K., Beavers S., Chen T., Christian K., Cooper M., Dufficy D., Gershman M., Glenshaw M., Hall A., Holzbauer S., Huang A., Langer A., Moore Z., Patel A.S., Carpenter L.R., Schaffzin J., Su J., Trevino I., Weiser T., Wiersma P., Lorick S., Verani J.R. Acanthamoeba keratitis multiple states, 2005-2007 MMWR [CDC web site]. Available in: http://www.cdc.gov/mmwr/. Accessed October 20, 2010.

Carlesso A.M., Artuso G.L., Simonetti A.M., Rott M.B. 2007. Isolation and identification of potentially pathogenic free-living amoebae in samples from environments in a public hospital in the city of Porto Alegre, Rio Grande do Sul. Revista da Sociedade Brasileira de Medicina Tropical, 40, 316-320. DOI: 10.1590/S0037-86822007000300013.

Caumo K., Frasson A.P., Pens C.J., Panatieri L.F., Frazzon A.P., Rott M.B. 2009. Potentially pathogenic Acanthamoeba in swimming pools: a survey in the southern Brazilian city of Porto Alegre. Annals of Tropical Medicine and Parasitology, 103, 477-485. DOI: 10.1179/136485909X451825.

Caumo K., Rott MB. 2011. Acanthamoeba T3, T4 and T5 in swimming-pool waters from Southern Brazil. Acta Tropica, 117 233-235. DOI: 10.1016/j.actatropica.2010.12.008. 
Cohen E.J., Buchanan H.W., Laughrea P.A., Adams C.P., Galentine P.G., Visvesvara G.S., Folberg R., Arentsen J.J., Laibson P.R. 1985. Diagnosis and management of Acanthamoeba keratitis. American Journal of Ophthalmology, 100, 389-395.

Corsaro D., Venditti D. 2010. Phylogenetic evidence for a new genotype of Acanthamoeba (Amoebozoa, Acanthamoebida). Parasitology Research, 107, 233-238. DOI: 10.1007/s00436-0101870-6.

De Carli G.A., Moura H. 2007. Amebas de Vida Livre. In: De Carli GA. 2nd Ed. Parasitologia Clínica, Seleção de Métodos e Técnicas de Laboratório para o Diagnóstico das Parasitoses Humanas. Atheneu, Rio de Janeiro, 417-427.

De Jonckheere J.F. 2003. Epidemiological typing of Acanthamoeba strains isolated from keratitis cases in Belgium. Bull Societ Belge Ophtalmology, 287, 27-33.

Gast R.J. 2001. Development of Acanthamoeba-specific reverse dotblot and the discovery of a new ribotype. Journal of Eukaryotic Microbiology, 48, 609-615.

Hiti K., Walochnik J., Haller-Schober E.M., Faschinger C., Aspöck H. 2002. Viability of Acanthamoeba after exposure to a multipurpose disinfecting contact lens solution and two hydrogen peroxide systems. British Journal of Ophthalmology, 86, 144 146. DOI: $10.1136 /$ bjo.86.2.144.

Iovieno A., Oechsler R.A., Ledee D.R., Miller D., Alfonso E.C. 2010. Drug-resistant severe Acanthamoeba keratitis caused by rare T5 Acanthamoeba genotype. Eye \& Contact Lens, 36, 183 184. DOI: 10.1097/ICL.0b013e3181da2350.

Khan N.A., Jarrol E.L., Paget T.A. 2002. Molecular and physiological differentiation between pathogenic and nonpathogenic Acanthamoeba. Current Microbiology, 45, 197-202. DOI: 10.1007/s00284-001-0108-3.

Lipener C., Ray C.B. 2008. Sistemas atuais de cuidado e manutenção de lentes de contato. Arquivos Brasileiros de Oftalmologia, 71, 9-13.

Maghsood A.H., Sissons J., Rezaian M., Nolder D., Warhurst D., Khan N.A. 2005. Acanthamoeba genotypes T4 from the UK and Iran and isolation of the T2 genotype from clinical isolates. Journal of Medical Microbiology, 74, 755-759. DOI: 10.1099/jmm.0.45970-0.

Marciano-Cabral, Cabral G. 2003. Acanthamoeba spp. as agents of diseases in humans. Clinical Microbiology Reviews, 16, 273 307. DOI: 10.1128/CMR.16.2.273-307.2003.

McClellan K., Coster D.J. 1987. Acanthamoebic keratitis diagnosed by paracentesis and biopsy and treated with propamidine. British Journal of Ophthalmology, 71, 734-736.

Molmeret M., Horn M., Wagner M., Santic M., Abu Kwaik Y. 2005. Amoebae as training grounds for intracellular bacterials pathogens. Applied and Environmental Microbiology, 71, 2028. DOI: 10.1128/AEM.71.1.20-28.2005.

Morlet N., Duguid G., Radford C., Matheson M., Dart J. 1997. Incidence of Acanthamoeba keratitis associated with contact lens wear. Lancet, 350, 414-416.
Patel A., Hammersmith K. 2008. Contact lens-related microbial keratitis: recent outbreaks. Current Opinion in Ophthalmology, 19, 302-306. DOI: 10.1097/ICU.0b013e3283045e74.

Pens C.J., da Costa M., Fadanelli C., Caumo K., Rott M. 2008. Acanthamoeba spp. and bacterial contamination in contact lens storage cases and the relationship to user profiles. Parasitology Research, 103, 1241-1245. DOI: 10.1007/s00436-0081120-3.

Radfort C.F., Minassian D.C., Dart J.K.G. 2002. Acanthamoeba keratitis in England and Wales: incidence, outcome, and risks factors. Britsh Journal of Ophthalmology, 86, 536-542. DOI: 10.1136/bjo.86.5.536.

Schaumberg D.A., Snow K.K., Dana M.R. 1998. The epidemic of Acanthamoeba keratitis: where do we stand? Cornea, 17, 310.

Shoff M., Rogerson A., Schatz S., Seal D. 2007.Variable responses of Acanthamoeba strains to three multipurpose lens cleaning solutions. Investigative Ophthalmology \& Visual Science, 84, 202-207. DOI: 10.1097/OPX.0b013e3180339f81.

Shoff M.E., Joslin C.E., Tu E.Y., Kubatko L., Fuerst P.A. 2008. Efficacy of contact lens systems against recent clinical and tap water Acanthamoeba isolates. Cornea, 27, 713-719. DOI: 10.1097/QAI.0b013e31815e7251.

Spanakos G., Tzanetou K., Miltsakakis D., Patsoula E., MalamouLada E., Vakalis N.C. 2006. Genotyping of pathogenic Acanthamoeba isolated from clinical samples in Greece - report of a clinical isolate presenting T5 genotype. Parasitology International, 55, 147-149. DOI: 10.1016/j.parint.2005.12.001.

Stothard D.R., Schroeder-Diedrich J.M., Awwad M.H., Gast R.J., Ledee D.R., Rodriguez-Zaragoza S., Dean C.L., Fuerst P.A., Byers T.J. 1998. The evolutionary history of the genus Acanthamoeba and identification of eight new 18S rRNA gene sequence types. Journal of Eukaryotic Microbiology, 45, 45-54. DOI: 10.1111/j.1550-7408.1998.tb05068.x.

Theodore F.H., Jakobiec F.A., Juechter K.B., Ma P., Troutman R.C., Panq P.M., Iwamoto T. 1985.The diagnostic value of a ring infiltrate in acanthamoebic keratitis. Ophthalmology, 92, 1471-1479.

Walochnick J., Obwaller A., Aspock H. 2000. Correlations between morphological, molecular, biological and physiological characteristics in clinical and nonclinical isolates of Acanthamoeba spp. Applied and Environmental Microbiology, 66, 4408-4413. DOI: 10.1128/AEM.66.10.4408-4413.2000.

Yu H.S., Kong H.H., Kim S.Y., Hahn Y.H., Hahn T.W., Chung D.I. 2004. Laboratory investigation of Acanthamoeba lugdunensis from patients with keratitis. Investigative Ophthalmology \& Visual Science, 45, 1418-1426. DOI: 10.1167/iovs.03-0433.

Zheng X., Uno T., Goto T., Zhang W., Hill J.H. 2004. Pathogenic Acanthamoeba Induces Apoptosis of Human Corneal Epithelial cells. Japanese Journal of Ophthalmology, 48, 23-29. DOI: 10.1007/s10384-003-0018-y. 